\title{
Bibnum
}

Textes fondateurs de la science

Mathématiques | 2018

\section{Liouville, le découvreur des nombres transcendants}

\section{Michel Mendès France}

\section{OpenEdition \\ Journals}

Édition électronique

URL : https://journals.openedition.org/bibnum/668

DOI : 10.4000/bibnum.668

ISSN : 2554-4470

\section{Éditeur}

FMSH - Fondation Maison des sciences de l'homme

\section{Référence électronique}

Michel Mendès France, «Liouville, le découvreur des nombres transcendants », Bibnum [En ligne],

Mathématiques, mis en ligne le 01 novembre 2008, consulté le 04 février 2023. URL : http:// journals.openedition.org/bibnum/668; DOI : https://doi.org/10.4000/bibnum.668

\section{(c) (†) (?)}

Creative Commons - Attribution - Partage dans les Mêmes Conditions 4.0 International - CC BY-SA 4.0 https://creativecommons.org/licenses/by-sa/4.0/ 


\section{Liouville, le découvreur des nombres transcendants}

par Michel Mendès France

Professeur émérite de mathématiques à I'Université de Bordeaux

En deux notes de 1844 aux comptes rendus de I'Académie des Sciences, Joseph Liouville établit l'existence des nombres transcendants.

Qu'est-ce qu'un nombre transcendant ? Un nombre $x$ est dit algébrique s'il est solution d'une équation polynomiale de type :

$$
f(x)=a x^{n}+b x^{n-1}+\ldots+g x+h
$$

où $a, b, \ldots ., g, h$ sont des entiers donnés non tous nuls. Ainsi, par exemple, les nombres suivants sont algébriques :

$$
-5,7 / 3, \sqrt{10}, \sqrt[3]{5-\sqrt{2}}, \sqrt{-1}
$$

Si le polynôme ne peut se factoriser en deux polynômes, $n$ s'appelle le degré du nombre algébrique. Les nombres de degré algébrique 1 correspondent exactement aux nombres rationnels. Les autres nombres algébriques sont dits irrationnels : dans la liste ci-dessus, les degrés respectifs sont 1, 1, 2, 6, 2.

Avant Liouville, on pouvait croire que tous les nombres étaient algébriques. Après lui, on savait qu'il en existait d'autres : un nombre non algébrique est dit transcendant.

\section{@@@@@@@}

Liouville donne deux preuves de l'existence de tels nombres ; toutes deux s'appuient sur la théorie des fractions continues pour établir le résultat fondamental suivant :

Si $x$ est un nombre algébrique réel de degré $\mathrm{n} \geq 2$ (donc irrationnel), alors il existe une constante positive non nulle $C$ telle que, pour tout nombre rationnel

$$
p / q \text {, on } a:\left|x-\frac{p}{q}\right|>\frac{C}{q^{n}} \text {. }
$$

Autrement dit, même si l'ensemble des rationnels est dense, un nombre algébrique irrationnel se laisse mal approcher par les rationnels. 
L'inégalité de Liouville peut s'établir de façon élémentaire sans faire appel aux fractions continues, que Liouville utilise: c'est pourquoi nous nous écarterons de cette partie de son texte, néanmoins pour aboutir au même résultat. Voici une preuve de cette inégalité. Le théorème des accroissements finis - bien connu de tout étudiant en première année de mathématiques à l'université - affirme que pour toute fonction réelle continue et dérivable $f$, il existe un nombre $t$ compris entre $x$ et $p / q$ tel que :

$$
f(x)-f\left(\frac{p}{q}\right)=\left(x-\frac{p}{q}\right) f^{\prime}(t) .
$$

Si donc $x$ est réel algébrique, il existe un polynôme à coefficients entiers non tous nuls tel que $f(x)=0$, et l'égalité précédente implique :

$$
x-\frac{p}{q}|=| \frac{f\left(\frac{p}{q}\right)}{f^{\prime}(t)}|=| \frac{q^{n} f\left(\frac{p}{q}\right)}{q^{n} f^{\prime}(t)} \mid \text {. }
$$

Nous allons chercher à minorer cette quantité par une quantité de type $A / q^{n}$ (inégalité de Liouville).

\section{Démonstration de l'inégalité de Liouville}

Cherchons d'abord à minorer le numérateur $q^{n} f(p / q)$. Comme le fait remarquer Liouville, cette quantité, qu'il appelle $f(p, q)$, est égale à $a p^{n}+b p^{n-1}+\ldots .+h q^{n}: c^{\prime}$ est un nombre entier.

Liouville avait pris la précaution d'indiquer que le polynôme $f$ avait été « débarrassé de tout facteur commensurable», c'est-à-dire réduit à la forme où il n'admet que des solutions irrationnelles. Cette précaution prise, le numérateur ne peut jamais être nul ; s'agissant d'un nombre entier (positif ou négatif), sa valeur absolue est toujours minorée par 1 .

Cherchons à présent à majorer la quantité $\left|\mathrm{f}^{\prime}(\mathrm{t})\right|$ figurant au dénominateur, en rappelant que $t$ est compris entre $p / q$ et $x$. On choisit $\mathrm{p} / \mathrm{q}$ tel que $-1<\mathrm{x}-\mathrm{p} / \mathrm{q}<1$. Alors, $\mathrm{f}^{\prime}$ étant une fonction polynomiale bornée sur l'intervalle $[x-1, x+1]$, elle prend sur ce segment des valeurs finies, qu'on peut majorer par une quantité 1 constante : $\left|f^{\prime}(t)\right|<A$.

En reportant ci-dessus, après avoir minoré le numérateur et majoré le dénominateur (avec $C=1 / A$ ), on obtient l'inégalité de Liouville : 


$$
x-\frac{p}{q}|=| \frac{f\left(\frac{p}{q}\right)}{f^{\prime}(t)}|=| \frac{q^{n} f\left(\frac{p}{q}\right)}{q^{n} f^{\prime}(t)} \mid>\frac{c}{q^{n}}
$$

@@@@@@@

Une fois établie cette inégalité, Liouville mentionne rapidement en une phrase la façon dont il construit un nombre non algébrique («Nous citerons en particulier la série... »). Détaillons la deuxième partie de sa «découverte ». Il observe que le nombre

$$
y=\sum_{k=1}^{\infty} \frac{1}{10^{k !}} \text { (on prend } a=10 \text { dans I'exemple qu'il donne en fin d'article) }
$$

est « trop bien approché » par les sommes partielles $\sum_{\mathrm{k}=1}^{\mathrm{N}} \frac{1}{10^{\mathrm{k} !}}=\frac{\mathrm{p}_{\mathrm{N}}}{10^{\mathrm{N} !}}=\frac{\mathrm{p}_{\mathrm{N}}}{\mathrm{q}_{\mathrm{N}}}$, d'où il conclura que y est transcendant. Détaillons ce calcul.

\section{La série de Liouville est un nombre transcendant}

Examinons la quantité $y-\sum_{k=1}^{N} \frac{1}{10^{k !}}=\sum_{N+1}^{\infty} \frac{1}{10^{k !}}=0,0000 \ldots \ldots .01 \ldots \ldots .$. le premier 1 apparaît à la position $(\mathrm{N}+1)$ ! après la virgule, et d'autres 1 apparaissent, de plus en plus espacés. On peut donc majorer cette quantité par exemple par le nombre où 2 apparaît à la position $(\mathrm{N}+1)$ ! après la virgule, suivi de 0 :

$$
\sum_{N+1}^{\infty} \frac{1}{10^{k !}}<\frac{2}{10^{(N+1) !}}=\frac{2}{10^{N !(N+1)}}=\frac{2}{\left(q_{N}\right)^{N+1}}
$$

Pour toute quantité positive non nulle $C$, cette dernière quantité est majorée $\operatorname{par} \frac{C}{\left(q_{N}\right)^{n}}$ dès lors que $N$ est assez grand pour que $q_{N}{ }^{(N+1-n)}>\frac{2}{C}$ (rappel : $q_{N}$ $=10^{N !}$ ). Donc, pour tout $\mathrm{n}$ donné, pour toute quantité positive non nulle $\mathrm{C}$, on a pu trouver une infinité de $\mathrm{p} / \mathrm{q}$ (à savoir les $\mathrm{p}_{\mathrm{N}} / \mathrm{q}_{\mathrm{N}}$ pour $\mathrm{N}$ assez grand) tels que $\left|y-\frac{p}{q}\right|<\frac{C}{q^{n}}$.

Ceci contredit l'inégalité de Liouville et permet de conclure que y est transcendant. On notera au passage qu'un nombre algébrique irrationnel ne peut être «approché de trop prés » par des nombres rationnels (inégalité de Liouville), en revanche certains nombres transcendants peuvent être ainsi « approchés ». 


\section{@@@@@@@}

Bien des années plus tard, Georg Cantor montre que «presque tous » les nombres sont transcendants, ce qui est évidemment assez surprenant puisqu'on est bien plus familiarisé avec les racines d'équation polynomiales ! En 1873, Charles Hermite établit la transcendance de $e$, puis suivant des idées assez proches, Ferdinand Lindemann montre en 1882 que $\pi$ est transcendant. Ce dernier résultat règle une fois pour toutes le vieux problème de la quadrature du cercle! On se convaincra en effet qu'à partir de la longueur unité, toute construction avec la règle et le compas ne peut donner que des nombres algébriques (d'ailleurs de degré $1,2,4,8,16$, etc. - théorème de Wantzel). Si la quadrature du cercle était possible, $\pi$ serait algébrique, ce qui est absurde.

Depuis, bien des familles de nombres transcendants ont été mises à jour. Si $a \neq 0$ ou 1 , et si $b$ est irrationnel, tous deux algébriques, alors $a^{b}$ est transcendant (A.O. Gelfond et Th. Schneider, 1934). En particulier, puisque $\mathrm{e}^{\pi}=$ $(-1)^{(-i)}$, on voit que $e^{\pi}$ est transcendant. En 1955, K.F. Roth améliore I'inégalité de Liouville et montre que pour tout $\mathrm{x}$ algébrique irrationnel, pour tout $\varepsilon$ positif, il existe une infinité de $p / q$ irréductibles tels que $|x-p / q|>1 / q^{2+\varepsilon}$. Ceci lui vaudra la très convoitée médaille Fields en 1958.

La théorie des nombres transcendants est aujourd'hui florissante, sans doute grâce aux beaux travaux du mathématicien Alan Baker dans les années 1960-70, pour lesquelles il recevra lui aussi la médaille Fields (1970).

La morale de cette histoire est, me semble t-il, que les deux notes de Liouville, quoique parfaitement élémentaires, sont d'une grande profondeur. Seul un grand mathématicien peut découvrir des idées simples et néanmoins très riches. 